\title{
Geografía y medio ambiente: Perspectivas de análisis
}

\author{
Jorge Andrés Rivera* \\ Magda Liliana Pérez**
}

Resumen. Este artículo explora la relación existente entre la geografía y medio ambiente a partir de un breve análisis histórico desde finales del siglo XVIII que intenta revisar las diferentes tendencias conceptuales con relación al estudio de los problemas ambientales. En este sentido, se espera evidenciar que el medio ambiente ocupa un gran lugar dentro del trabajo geográfico y su objeto de estudio, sin pretender ninguna patente o sello de pertenencia exclusivo de esta disciplina. Por el contrario, la discusión muestra el diálogo con otras ciencias en lo tocante a la cuestión ambiental. No obstante, se aspira a mostrar el aporte de la geografía a este tópico con base en la manera como emprende el análisis (escalas, dominio de síntesis, etc). Palabras clave: Relación Hombre Medio, Naturaleza $\sim$ Sociedad; relación Geografía filosofía Ambiente (Determinismo, posibilismo, antropocentrismo; etc.); ecología, ecosistemas, ecocentrismo, ecofeminismo, etc.

* Administrador Ambiental, Universidad Tecnológica de Pereira. Alumno del Programa de Estudios de Maestría en Geografía del Convenio UPTC-IGAC.

** Ingeniera Catastral y Geodesta. Especialista en Sistemas de Información Geográfica, Universidad Distrital Francisco José de Caldas. Alumna del Programa de Estudios de Maestría en Geografía del Convenio UPTC-IGAC. Investigadora del grupo de investigación GICOGE, reconocido por Centro de Investigación y Desarrollo Científico (CIDC) de la Universidad Distrital.

Los autores desean agradecer por su colaboración en la elaboración y corrección del presente artículo a los profesores Jaime Bonilla y Ovidio Delgado. 


\section{Introducción}

Para abordar y analizar el papel de la geografía en el estudio de la problemática ambiental se debe comprender inicialmente el desarrollo conceptual de la disciplina en torno a esta problemática, teniendo en cuenta que los fundamentos teóricos y metodológicos como la práctica investigativa, educativa y la toma de decisiones sobre el tema son el resultado del contexto social, económico, político, cultural, histórico y ambiental de la sociedad donde éstas se inscriben. De esta forma, es importante anotar que las diferentes perspectivas conceptuales sobre esta temática están influenciadas por diversas corrientes de pensamiento filosófico, que a su vez han venido siendo rebatidos o sustituidos total o parcialmente por otros nuevos, pero que también son en algunas oportunidades retomados y redefinidos.

Para este fin, se plantean dos preguntas o núcleos problemáticos a desarrollar a través de este artículo. El primero es, ¿Cuáles han sido los aportes realizados por las diferentes corrientes geográficas en la construcción de una epistemología ambiental?; el segundo, ¿Cuáles son los problemas geográfico-ambientales concretos y cómo ha participado la geografia en su resolución?

Para responder la primera pregunta, se analiza la relación hombre-medio a través de una aproximación al pensamiento geográfico-ambiental, sin dejar de lado las complementariedades y aportaciones de otras disciplinas. Para este fin se citan los trabajos y las contribuciones conceptuales realizadas por diferentes geógrafos que han ayudado ha nutrir dicha discusión desde finales del siglo XVIII hasta la época actual; posteriormente se define la relación entre el discurso geográfico y las diferentes visiones ambientalistas. Para responder al segundo interrogante, se exponen algunas de los problemas ambientales que caracterizan a las sociedades actuales y cómo la geografía ha buscado a partir de aplicaciones prácticas, dar resolución a los mismos. Finalmente, a manera de conclusión, se presenta la reflexión sobre la especificidad de la geografía en el análisis ambiental, teniendo en cuenta las unidades de análisis y los dominios de síntesis.

\section{Relación hombre-medio: aproximación al pensamiento geográfico-ambiental}

Para iniciar, es pertinente hacer referencia a las distintas maneras como la ciencia geográfica ha contemplado la relación entre el hombre y su medio. La producción conceptual desarrollada sobre dicha relación tiene una base filosófica muy fuerte, en tanto se fundamenta en su objeto de estudio, es decir, en el análisis de la posición del hombre en el universo. Dentro de los enfoques que han soportado diferentes miradas sobre la dialéctica 
sociedad-naturaleza, se encuentran las cosmovisiones indígenas ${ }^{1}$, orientales ${ }^{2}$ y las de occidente, entre otras; sin embargo, esta última es la que se ha extendido y afianzado con mayor notoriedad por constituirse en el pilar del sistema político, socio-económico, cultural y académico dominante, construido sobre la base de la tradición religiosa judeo-cristiana y la racionalidad que dieron origen a concepciones como la de una tierra diseñada para la especie humana ${ }^{3}$, donde el medio es visto como la materia prima e instrumento más eficiente para alcanzar los fines del hombre (progreso, desarrollo, perfección y dominio).

Otro tópico considerado dentro de esta perspectiva occidental es el estudio de la influencia del ambiente en la cultura. Este concepto se deriva inicialmente del contraste entre la naturaleza y las costumbres en diferentes lugares, llegando a ser usado en la interpretación del orden de la cultura humana y las diferencias biológicas. Esto no es totalmente incompatible con la idea de una tierra diseñada, lo cual atrajo a personalidades como Thomas Malthus (1766-1834), quien enfatizó no solamente en la influencia de diferentes ambientes sino también en las limitaciones que la tierra impone al desarrollo social. Malthus ayudó a mantener viva una idea de gran antigüedad que resurgió de nuevo en el siglo XIX como determinismo ${ }^{4}$.

Dentro de la geografía, Carl Ritter (17791859), retomando las ideas maltusianas afirmó que el ambiente físico era capaz de determinar el curso del desarrollo humano. Su ideología se vio validada por la publicación del Origen de las Especies de Charles Darwin en 1859, en donde se resaltaba la estrecha relación entre los organismos y sus hábitats, y la noción de presión de la selección natural. Esta obra dio luces para sustentar las bases de la cientificidad que aspiraba la geografía ${ }^{5}$, y en particular el determinismo ambiental, influenciando a geógrafos como Freidrich Ratzel (1844-1904) y Ellen Churchill Semple (1863-1932).

Ratzel tomó la idea de unidad de vida orgánica, y la analogía orgánica de Spencer en la noción de una geopolítica del Estado. Según él, cada organismo viviente requiere un territorio, es decir, su espacio vital (lebensraum). La

\footnotetext{
1 Dentro de los enfoques míticos y tradicionales de las comunidades indígenas se comparte un respeto y armonía en sus tareas cotidianas con la Tierra, la cual posee una connotación de "madre", portadora de vida. Las comunidades en su esencia más íntima están organizadas, creadas y animadas por ella y de una extensa red de relaciones que las une no solo a su entorno o espacio geográfico (biosfera, litosfera, atmósfera) sino también con la historia constitutiva y de formación de los procesos que dieron origen a la vida.

2 Las reflexiones filosofias orientales no giran únicamente en torno a las relaciones humanas, los valores morales y el gobierno; este es sólo uno de los aspectos de su pensamiento. Complementario a éste, está el lado místico de su carácter, que exige que el objetivo más importante de la filosofía sea trascender el mundo de la sociedad y la vida cotidiana y llegar así a un plano superior de conciencia, donde el ideal oriental del hombre iluminado (sabio) es lograr una unión mística con el universo.

3 Esta característica se puede justificar, en las posiciones y argumentaciones realizadas en: GLACKEN, Clarence. Traces on the Rhodian Shore. Nature and Culture in Western Thought from ancient times to the end of the Eighteenth Century. Berkeley: University of California Press. 1967.

4 DARWIN, Charles. Essay on the Principle of Population. 1798.
} 
generalización de estas ideas fueron interpretadas para servir a los intereses del imperialismo en varios aspectos, como la adquisición de territorios, explotación económica, militarismo, prácticas de clase y dominación de razas [Peet, 1998].

Posteriormente, interpretando las ideas de Ratzel, Semple pensó que las regiones de la tierra producían gente con diferentes temperamentos; de este modo describió a los europeos del norte como personas más inteligentes y vigorosas, mientras que los europeos del sur eran calificados como personas despreocupadas, emocionales y soñadoras; cualidades que según la autora, entre los negros del cinturón ecuatorial degeneraron en graves taras raciales [Peet, 1998].

De igual manera, Ellsworth Huntington (1876-1947), trató de buscar una evidencia objetiva del efecto del ambiente físico, y en particular el clima, el cual él consideraba una importante influencia en el comportamiento humano [Huntington, 1915]. Su hipótesis consistía en demostrar una relación causal entre ciertos climas templado-fríos, que estimulaban la actividad humana y las elevadas civilizaciones actuales del Noreste de Europa. Huntington sugería que los mejores climas para trabajar eran aquellos en donde existían variedades y en los cuales las temperaturas caían dentro de cierto rango ${ }^{6}$. De esta forma, se puede deducir que él también estableció una relación entre ambiente físico, clima y comportamiento humano [Huntington, 1929].

Con ideas similares a las de Huntington; Griffith Taylor (1880-1963), fue aún más cuidadoso en el momento de reunir correctamente datos acerca del medio ambiente y relacionarlos con sus ideas de habitabilidad humana. Taylor finalmente sugirió que aunque el ambiente físico guía a las sociedades en direcciones particulares, ellas pueden controlar su influencia [Taylor, 1927].

En esta dirección epistemológica se puede manifestar que la concepción del determinismo geográfico-ambiental abanderada por Ratzel dentro de la disciplina, y expuesta claramente en su obra, Antropogeografía soslayaba otro tipo de explicaciones causales, que tienen una incidencia significativa en el

\footnotetext{
5 En este momento histórico, es oportuno plantear que la geografía se estaba apenas consolidando como una ciencia universitaria, por esta razón, la disciplina en su intento por el reconocimiento dentro de este mundo académico optó como método de análisis imperativo de la relación hombre-medio y la dialéctica humanización-naturalización el de las ciencias naturales, enfatizando su actitud positivista, soportada en los principios de la ciencias empírico analíticas. Por consiguiente, hay que resaltar la gran influencia de la biología y la ecología en la geografía, y en particular de la explicación de fenómenos geográfico-ambientales a partir del concepto de sistema, tanto desde el punto de vista metodológico como del empírico. Esta perspectiva de análisis sirvió como punta de lanza para extender el método de las ciencias naturales a la explicación de las relaciones entre la sociedad y la naturaleza en términos de homeostasis, adaptación, flujos y/o ciclos de energía, etc.

6 Con el propósito de verificar su posición ideológica y entender mejor la verdadera relación entre el hombre y su ambiente, este autor analizó el efecto de espacios geográficos "extremos", tomando como caso de estudio el desierto de Kalahari en el suroeste de África y las formas de vida, sustento y nivel de civilización alcanzado por la tribu Bushmen. HUNTINGTON, Ellsworth. The Human Habitat. United States of America: Plimpton Press, 1929. P. $35-47$.
}

140 Jorge Andrés Rivera - Magda Liliana Pérez 
comportamiento, decisiones y actividades sociales en relación con su medio (véase pie de página 1 y 2 ).

La razón por la cual se explicó la distribución espacial, la capacidad intelectual y el desarrollo material de las diferentes culturas y razas en función del medio físico desde el determinismo ambiental obedecía más que a un carácter académico a un interés social, política y económico de los países imperialistas por expandir sus economías explotando y avasallando territorios extranjeros.

La proclamación de otro paradigma ${ }^{7}$ del estudio de la relación hombre-medio surgió de la corriente francesa en rechazo a los argumentos deterministas, afirmando que era inútil oponer el entorno natural del hombre a su medio social, justificando el predominio del primero sobre el segundo (Vidal de la Blache).

Vidal de la Blache (1845-1918), decía que no era razonable establecer límites entre los fenómenos naturales y culturales, y que ambos deberían ser considerados como un todo inseparable. En oposición al determinismo ambiental, desarrolló el concepto de "posibilismo" según el cual el medio físico limita la actividad humana, pero sin determinarla, a la vez que el ser humano influye sobre su entorno ${ }^{8}$ empleando su capacidad intelectual y creativa en la adecuación de diferentes espacios para sus sistemas productivos.

En la conclusión de su trabajo planteaba que el vínculo del hombre con su medio, por muy esencial que fuera, era lo suficientemente complicado y múltiple para que el geógrafo no cayera en el determinismo. Para él, el medio que el geógrafo debía estudiar era una "región determinada", en donde se reflejaban identidades económicas, sociales, ideológicas y psicológicas plasmadas en diversos paisajes, como expresión de una sociedad dinámica que se conjugaba con la naturaleza. No obstante, aunque sus planteamientos se definieron como apolíticos, en la actualidad desde la óptica de la geografía radical se ha manifestado la intención del posibilismo en ayudar a las aspiraciones y necesidades de la burguesía francesa que buscó nuevas fuentes de riqueza económica en su proyecto colonialista, tal como lo hizo en su momento la escuela alemana con el determinismo ambiental.

No obstante, dentro de esta perspectiva de análisis, no se puede pasar por alto el aporte de Elisée Reclus, quien puede ser visto como el precursor del pensamiento bioregional. Él concluyó que nosotros y nuestras culturas somos reflejo de la tierra

Para Khun paradigma es: "una realización científica que goza de reconocimiento universal y logra validez durante cierto tiempo, periodo en el cual proporciona un modelo de problemas y soluciones compartidos por una comunidad científica”. KHUN, Thomas. Estructura de las Revoluciones Científicas. 1962

8 Para Vidart entorno es: "todo el ámbito de lo visible, de lo alcanzable, de lo relacionable con el hombre y con los organismos. Este es el más amplio, genérico y neutral de los círculos concéntricos del espacio geográfico. El entorno esta incluido en el espacio geográfico del planeta, pero es más que este, pues además de abarcar las estructuras espaciales y a sus relaciones, envuelve las relaciones funcionales ecosistémicas y antroposistémicas. VIDART, Daniel. Filosofía Ambiental. Bogotá: Editorial Nueva América, 1986. 
y de regiones específicas del planeta en el cual nos hemos desarrollado. De hecho; según Clark ${ }^{9}$, él no sólo fue la figura pionera de la geografía social sino además de la teoría ecológica social. Esta teoría ha llegado a ser tema central de la filosofía ambiental y de la ética ambiental actual. Uno de los más importantes aspectos de su teoría es la concepción holística, la interpretación dialéctica del lugar de la humanidad en el mundo natural. Reclus, demostró que la Tierra es un planeta viviente donde las acciones humanas tienen efectos negativos y/o positivos, y estos dependen, entre otros, del sistema político y económico dentro de los cuales tienen lugar (La terre).

Dentro de esta corriente de pensamiento francés, Jean Brunhes (1869-1930), afirmó que los geógrafos debían estudiar siempre la acción del hombre sobre la naturaleza sin separarla jamás del estudio de la Geografía natural o física. En este sentido, argumentó que: "existen unos vínculos de interdependencia $\mathrm{y}$ repercusión por los cuales los hechos económicos, sociales y políticos de la población y de la civilización material se unen con los de la naturaleza física"10. De igual manera, Lucien Febvre (18781956), estaba en contra del determinismo, sustentando su teoría en la movilidad del hombre y su capacidad de poner al medio ambiente como un agente pasivo. Este autor canalizó las ideas de Brunhes y de La Blanche, enunciando que: "no existen necesidades, sino posibilidades por todas partes; y el hombre como maestro de las posibilidades corresponde decidir sobre su uso"11. Finalmente, H. J. Fleure (18771969), fue quien trató de formular regiones del mundo con base en características humanas aportando una visión diferente a las regiones bióticoclimáticas tradicionales. Es así como Fleure, planteó un esquema en el cual incluía «regiones de esfuerzo», «regiones de hambre» y «regiones industrializadas $\rangle^{12}$. Sin embargo, en razón a las debilidades que encontró en su esquema a partir de un proceso autocrítico, observó la importancia de la influencia que ejercía el medio físico ${ }^{13}$.

Esta última argumentación pone de manifiesto que la geografía regional no pudo, en términos generales, romper con la visión determinística; al igual que sigue sustentando su racionalidad positivista, instrumental y técnica en favor del sistema económico, político y social dominante.

Surge luego una corriente que es conocida como geografía Cultural, la cual se caracterizó por tener al paisaje como centro o foco de análisis, pues ahí convergen la naturaleza y la acción humana. En Alemania, Pasargue fue su principal cultivador; diez años más tarde,

\footnotetext{
9 CLARK, John. The dialectical Social Geography of Elisée Reclus. Ed. Rowman \& Littlefield Publishers, 1997.p.117.

10 BRUHNES, J. Human Geography. New York, 1925.

11 FEBVRE, L. A Geographical Introduction to History. Londres, 1925.

12 FLEURE, Herbert John. Human Regions. Scotland, 1919.

13 HARO, Juan. Calidad y Conservación del Medio Ambiente. Madrid: Ed. Cincel, 1983, p.13.
}

142 Jorge Andrés Rivera - Magda Liliana Pérez 
Carl Ortwin Sauer ${ }^{14}$ (1889-1975), se erigió como el más importante exponente de la geografía cultural, desarrollando la idea de ecología humana (resalta la relación entre factores humanos y físicos). La tarea fundamental de esta geografía era la de facilitar las claves para la explicación del paisaje y dirigir una certera mirada retrospectiva al reconstruir con rigor la evolución histórica de los paisajes ${ }^{15}$. Todo este cúmulo de influencias y reacciones llevaron a constituir la "Escuela Geográfica Cultural de Berkeley"16, en ésta el regionalismo se transforma en el estudio de paisajes culturales ${ }^{17}$.

Una de las obras que representan un interés especial es la de Pierre Gourou ${ }^{\mathbf{1 8}}$. Este autor sintetizó los anteriores aportes y supo entrever las tareas futuras que corresponderían a la geografía en la actual situación de deterioro del medio ambiente. Concluyó su obra afirmando que la acción paisajística del hombre puede ser degradante para el medio y nefasta para los propios intereses del hombre.

Posterior al trabajo desarrollado sobre la relación sociedad-naturaleza por la geografía regional y cultural surge un nuevo enfoque teórico de carácter cuantitativo $^{19}$, con el que se intentó desarrollar modelos que explicaran los patrones de organización social en función a la estructura espacial y el medio físico natural ${ }^{20}$. Para ello, se tomaron como ejes de análisis la ciudad considerándola como una "comunidad ecológica" y la "región". El objetivo fundamental de estos modelos era formalizar una teoría que permitiera optimizar la localización de sistemas productivos y de asentamientos humanos o poblacionales con relación a la ubicación y distribución de las materias primas y recursos naturales que proporcionaba el

14 Según Sauer: "el estudio del paisaje, y en particular de los fenómenos, la conexión entre ellos y su orden dentro de un área especifica, es una tarea científica, y a su parecer la única a la que la geografía debía dedicar su energía", [UNWIN, 1992. p., 142 ].

15 En este escenario la geografía cultural comparte ciertas inquietudes con el objeto de estudio de la antropología, aunque bastante distintas en foco a las de la disciplina geográfica (la forma como los grupos humanos crean, mantienen, refuerzan, perpetúan y promueven su cultura).

16 Aunque esta escuela gozó de buena aceptación dentro de la geografía americana en los años treinta y a principios de los cuarenta, la aparición de otra perspectiva de análisis y estudio de los paisajes abanderada por Hartshone, trató de relegar la compresión histórica, por considerarla poco objetiva y encontrarla fuera de los límites de la "ciencia", por el contrario hizo énfasis en un análisis contemporáneo (escala de tiempo presente); esta corriente fue la que tomo mayor fuerza dentro de la disciplina geográfica, hasta finales de los años cincuenta, [UNWIN, 1992. Pág., 149].

17 Como estudio representativo de esta escuela se encuentra el libro editado por William Thomas, Ilamado: "Man's Role in Changing the Face of the Earth". Chicago: University of Chicago Press, 1956.

18 GOUROU, Pierre. Los Países del Delta Tokinais. 1936.

19 Con este enfoque se intentó superar las críticas realizadas a la geografía, como lo enuncia Morill recordando la impresión que tenían de esta disciplina como "débil intelectual y numéricamente, y objeto de escasa estima por parte de otras disciplinas". De esta forma el propósito de los geógrafos que optaron por esta perspectiva era incorporarla a la corriente principal de la ciencia (de carácter neopositivista), desarrollando para ello teorías, verificándolas y tratando así de explicar la organización y evolución del paisaje, [UNWIN, 1992. Pág., 169].

20 Los conceptos y teorías que sustentan estos modelos interpretativos-explicativos provienen de disciplina como la economía (Von Thünen, Christaller, Lösch), la sociología (Escuela de Ecología Humana y Física Social) y la matemática (análisis estadístico, cibernética, etc) 
medio o espacio geográfico ${ }^{21}$. Sustentando los modelos y teorías se encontraba el concepto de sistema, el cual se convirtió en el elemento determinante para analizar y combinar los fenómenos humanos y ambientales. Esta integración del hombre y el medio en una estructura sistémica halló su expresión más clara en el desarrollo de la noción de ecosistema propugnado por biólogos como Odum ${ }^{22} \mathrm{y}$ geógrafos como Stoddart, éste último recomendó adoptar dicho concepto como marco conceptual para la investigación geográfica $^{23}$.

Sin embargo, al plantear la geografía dentro de un marco conceptual naturalista, se cayó nuevamente en un determinismo que sumergió a la disciplina en posturas reduccionistas a tal punto que se fundamentó la definición de categorías que diferenciaban las actividades agrícolas en función del medio ambiente ${ }^{24}$ y de su localización en la superficie terrestre (zona tropical, subtropical, etc), ${ }^{25}$ dejando de lado otro tipo de factores que determinan los sistemas agrícolas, como son las variables culturales, económicas, sociales y políticas entre otras.

El sistemismo pretendía elevar la geografía a una disciplina más atractiva y destacada, desconociendo los problemas metodológicos a los que se enfrentaba al pretender enlazar directamente, sin solución de continuidad epistemológica, las perspectivas regionales o corológicas de la geografía clásica, con las articulaciones conceptuales de la teoría general de sistemas. Este tipo de críticas a la nueva geografía positivista lógica, generaron como reacción dos posiciones contestatarias, la primera de ellas surgió hacia el inicio de los años sesenta como geografía radical, mientras la segunda aparece hacia finales de los sesenta como geografía humanística.

La geografía radical tomó como soporte conceptual y teórico los escritos de Marx, para establecer una reacción crítica a la crisis del capitalismo de la época, como lo fue el inicio de los movimientos a favor

\footnotetext{
${ }^{21}$ El espacio geográfico es el conjunto de estructuras espaciales y de relaciones entre ellas, que ocurren en la superficie de la Tierra como objeto de la acción, la dotación de sentido y la interpretación de los humanos. El espacio geográfico es, por tanto, una construcción social, ya como objetivación material de la cultura, ya como significación y simbolización, en el ámbito de las ideas y de la estética, [FLOREZ, MONTAÑEZ, 1997. Pág., 181]

22 ODUM, E. P. Ecology. New York, 1963.

23 Este autor señala la especial importancia de cuatro aspectos del ecosistema en la geografía: 1) El concepto agrupa elementos humanos, biológicos y zoológicos en una estructura única e integrada, 2) Los ecosistemas están estructurados con orden que facilita el estudio lógico por parte de los geógrafos, 3) Funcionan a través de un flujo continuo de entradas y salidas que pueden medirse, 4) En términos de los sistemas generales son ejemplos de sistemas abiertos que tienden al estado de reposo, [UNWIN, 1992. p., 181].

24 Medio ambiente es el marco en el cual se desarrolla la vida de un organismo. El organismo contribuye a conformar ese medio en que vive y a su vez es conformado. El ambiente tiene ingredientes naturales, humanos y humanizados. VIDART, Daniel. Filosofía Ambiental. Bogotá: Editorial Nueva América, 1986.

25 CHORLEY, Richard J., HAGGETT, Peter. La Geografía y los Modelos Económicos. Madrid: Instituto de Estudios de Administración Local, 1971, p. 399-401.
}

144 Jorge Andrés Rivera - Magda Liliana Pérez 
de los derechos civiles (ghettos y negritudes), la polémica guerra de Vietnam y los movimientos feministas ${ }^{26}$. La geografía radical tenía como objetivo llegar a la raíz de todos los problemas a estudiar, tales como la desigualdad, el racismo, el sexismo, la contaminación ambiental, etc. Muchos de los geógrafos que compartían sus principios se auto definían como una nueva geografía de izquierda.

El marco conceptual sobre el que se analizó la relación sociedad-naturaleza se baso en el concepto dialéctico de estructuración en el cual hay una interacción constante y recíproca entre el hombre y la naturaleza ${ }^{27}$; de igual forma se evidenció la introducción de la ecología política como nuevo marco de análisis e interpretación de la relación sociedad naturaleza; esta escuela puso énfasis en la relación existente entre los patrones de uso de los recursos y las fuerzas económicas y políticas en la sociedad.

De igual forma, en oposición a los esquemas planteados por el positivismo lógico, la geografía humanística inspirada en la fenomenología y el existencialismo, rechazó la objetividad de éste, sustituyéndola por un pensamiento subjetivo que le permitió a la disciplina geográfica replantear la dialéctica hombre-medio en función de los sentimientos, emociones y percepciones de los seres humanos con respecto al medio natural que habitan.

Dentro de la escuela geográfica humanística, Edward Relph afirmaba que el ser humano es el punto fundamental de referencia para todos los objetos y hechos de la naturaleza bien reconocidos por la geografía, pero además, hombre y naturaleza hacen parte de un sistema unificado por la necesidad del mismo, sus intenciones y su existencia. Relph sostenía, que el conocimiento de los lugares era esencial en la existencia humana. El lugar era concebido como un aspecto complejo de la experiencia humana en el mundo, por lo que la localización o la posición era un estado insuficiente de lugar. Existe una gran relación entre sociedad y lugar, en donde cada uno refuerza la identidad del otro ${ }^{28}$.

Yi-Fu Tuan incluyó el medio ambiente en sus concepciones humanísticas; para él, la geografía humanística debía observar las relaciones de las personas con la naturaleza, su comportamiento geográfico, así como sus sentimientos y sus ideas con respecto al espacio y al lugar ${ }^{29}$. En el concepto de Tuan la geografía humanística servía a la sociedad

26 Estos movimientos feministas generaron en la geografía un área de estudio (geografía de género), que supero la discusión dentro del enfoque marxista sobre la crítica teórica y filosófica para dar paso a la acción práctica. [UNWIN, 1992. p., 233].

27 Según Marx: "el trabajo es en primer lugar un proceso entre el hombre y la naturaleza, un proceso en el que el hombre mediante sus propias acciones, media, regula y controla el metabolismo entre él y la naturaleza. A través de este movimiento, el hombre actúa sobre la naturaleza exterior y la cambia, cambiando de este modo simultáneamente su propia naturaleza". [GREGORY, 1984. p., 177]

28 RELPH, Edward. Rational Landscapes and Humanistic Geography. Beckenham, 1981.

20 PEET, Richard. Modern Geographical Thought. Blackwell Publishers Editor, 1998. 
esencialmente para elevar su nivel de conciencia. Su obra más reconocida es "Topophilia" 30 , donde se encargó de explorar los lazos afectivos entre la gente y el lugar, haciendo énfasis en las maneras de percibir el medio ambiente; afirmaba que una persona es un organismo biológico, un ser social y un individuo, y que la percepción, la actitud y los valores reflejan los tres niveles del ser.

Posteriormente, se converge en los años ochenta a dos corrientes: el realismo y el postmodernismo. Según Unwin ${ }^{31}$, la primera pretendía alcanzar un metateoría global dentro de la cual pudiera comprenderse la diversidad filosófica en los últimos veinte años, el realismo era concebido como la posibilidad de unificar las diversas críticas que habían surgido en torno al positivismo y sus procedimiento metodológicos se basaban en la necesidad de identificar los mecanismos causales y las regularidades empíricas de fenómenos determinados en el espacio en un periodo o tiempo determinado. Según Bhaskar ${ }^{32}$ una de las principales ventajas del realismo, era la capacidad potencial de englobar las ciencias naturales y las ciencias sociales, al respecto Harré33 recalcó que por definición las estructuras sociales, a diferencia de las estructuras del mundo natural, no son independientes de los agentes humanos individuales, [Unwin, 1992].

El postmodernismo, por el contrario rechazaba la posibilidad de generar una metateoría que explicara todos los procesos sociales, y alentaba, en contraposición, una nueva mirada que permitiera interpretar los cambios y las contradicciones sociales en una escala local. De este modo, los primeros trabajos desarrollados por esta escuela geográfica trataban temas relacionados con el urbanismo y la planificación con un enfoque crítico basado en la teoría so$\mathrm{cial}^{34}$. Entre los geógrafos que han tratado de llevar a la práctica esta nueva perspectiva de análisis, el trabajo más representativo es el de Soja $(1989)^{35}$, en el cual a partir de una sucesión de miradas fragmentadas de carácter reflexivo e interpretativo, analizó el carácter específico y único del paisaje geográfico de la ciudad de Los Ángeles, observando la espacialidad fundamental de la vida social, las relaciones adhesivas entre la sociedad y el espacio, entre otras.

Finalmente, con base en la variedad de aproximaciones conceptuales y metodológicas desarrolladas y empleadas

\footnotetext{
so TUAN, Yi-Fu. Topophilia: A Study of Environmental Perception, Attitudes and Values. Englewood Cliffs, NJ: Prentice-Hall, 1974.

31 UNWIN, T. Op cit ., p. 244.

32 BHASKAR, R. Scientific Realism and Human Emancipation. Londres: Verso, 1986

33 HARRÉ, R. Varieties of Realism. Oxford: Basil Blackwell, 1986.

34 Para Dear, la mayor influencia del postmodernismo en la geografía se ve evidenciada en el trabajo de las siguientes temáticas: 1) Paisajes culturales y la construcción de lugar, 2) Los paisajes económicos del post-fordismo, 3) Las políticas del postmodernismo, el descontento de la geografía feminista con el postmodernismo y las preguntas sobre el postcolonialismo, 4) La construcción del individuo y su identidad, 5) Medio ambiente.

35 SOJA, E. W. Postmodern Geographies: the reassertion of space in critical social theory. Londres: Verso, 1989.
}

146 Jorge Andrés Rivera - Magda Liliana Pérez 
por la geografía durante el periodo referido, en torno a la relación hombremedio, la dialéctica humanizaciónnaturalización y los problemas ambientales, es oportuno señalar a continuación la relación entre estas concepciones geográficas y las diferentes visiones o enfoques ambientales; como también, exponer la manera como los geógrafos han utilizado las teorías y métodos de descripción, explicación, reflexión y predicción, en diferentes investigaciones, en la enseñanza de la temática ambiental, o en la resolución de los problemas ambientales que aquejan a las sociedades actuales, como son el cambio climático, el efecto invernadero, la perdida de biodiversidad, la contaminación hídrica, etc.

\subsection{Enfoques Ambientales}

El ambientalismo como un movimiento social ha sido fuente de fascinación para las ciencias sociales, de esta manera los geógrafos junto a los sociólogos, psicólogos sociales y politólogos entre otros, han sobresalido en este debate y han hecho importantes contribuciones para el entendimiento de los orígenes, la evolución y las ideologías del movimiento ambiental moderno.
No obstante, aunque es de amplia aceptación que el origen del movimiento ambiental moderno se da en los años 60 , las raíces ideológicas y filosóficas del ambientalismo tienen una historia mucho más larga. David Pepper $(1984)^{36}$, en una síntesis comprensiva, investiga diversas raíces en las ciencias clásicas en el siglo XIX, tales como el romanticismo ${ }^{37}$ y el conservasionismo ${ }^{38}$ en los escritos de Malthus y Darwin, así como en diversas ideologías políticas. Lowe y Goyder ${ }^{39}$, sostenían que la preocupación ambiental formaba parte de la tardía reacción intelectual Victoriana, a muchos de los principios del liberalismo económico. Grupos liderados por filósofos sociales como John Ruskin y William Morris dieron expresiones de inconformismo frente al industrialismo y el urbanismo, y se opusieron fuertemente a la destrucción de la herencia cultural y del mundo natural, el cual se soportaba en la continuidad, estabilidad y la tradición, en contra de la intranquilidad y el desarraigo que producía el movimiento industrial capitalista.

En cuanto a las concepciones geográficas que atienden al análisis de la relación hombre-medio a principios del siglo XX, el posibilismo ha alimentado e influenciado la orientación ideológica del

36 Pepper, David. The roots of modern environmentalism. Londres: Croom Helm, 1984.

37 Esta filosofía enfatiza en la interdependencia y relación entre los humanos y la naturaleza. En oposición al entendimiento de la naturaleza por parte de tradición judeo cristiana, los románticos creían que todas las criaturas -humanas y otras- poseían una presencia divina y por tal razón merecen respeto, [Knox, 2001, p.155].

38 El conservasionismo sostiene que los recursos naturales debían ser usados acertadamente, de forma que la relación entre la sociedad y el mundo natural no debe basarse en su explotación. [Knox, 2001, p.155].

39 LOWE, P.; GOYDER, J. Environmental Groups in Politics . London: Allen and Unwin, 1983. 
antropocentrismo ${ }^{40}$. En este enfoque el ambiente es considerado como un proveedor de servicios y soportes para la vida de la sociedad humana. Esta ideología esta fundamentada en leyes del desarrollo social que destacan el papel decisivo que tiene la organización social en el carácter y dinámica de las relaciones sociedad-naturaleza. Este enfoque coloca en primer plano la esencia social del hombre y tiende a minimizar los condicionantes "naturales" del desarrollo económico y social.

De la misma forma, Lowe y Goyder identificaron otras dos eras del ambientalismo y separaron particularmente dos periodos activos en la evolución del movimiento, uno de ellos se encontraba situado en el intermedio de la época de guerra (Primera y Segunda Guerra Mundial) en momentos en los que la conciencia social se fue ampliando y dando origen a nuevos grupos de presión $y$ en segundo lugar se encuentra el momento donde emerge el movimiento ambientalista entre los años 60 y principios de los 70 con algunos representantes de la geografía radical de género que cimentaron el ecofeminismo.

La geografía de género analizó y diferenció las relaciones y los roles que mantienen hombres y mujeres con la
Naturaleza, superando el discurso habitual que ha establecido un análisis del medio ambiente de carácter "macro" (incidencia de infraestructuras, contaminación industrial, incendios forestales, desertificación, etc.), donde las relaciones se establecen en función de las grandes actuaciones políticas y económicas, esto es, de las esferas controladas por los hombres, en cuanto detentadores del poder político y económico. El enfoque de género parte de un análisis "micro" como es la gestión diaria de los recursos por parte de cada individuo o pequeña comunidad, introduciendo temas tales como la incidencia ambiental del consumo diario de leña y agua en el Tercer Mundo, o la gestión de los residuos domésticos en los países desarrollados ${ }^{41}$.

La idea central del ecofeminismo, en cuanto a "feminismo ambientalista" consiste en plantear la existencia en las sociedades patriarcales de unas relaciones de poder de los hombres con respecto a las mujeres y a la naturaleza, de modo que el control y explotación de las mujeres y de la naturaleza tienen muchos elementos comunes; se afirma que "los problemas ambientales son un aspecto más del mismo injusto orden económico, social y cultural que oprime y explota a las mujeres", [Bru, 1993, p. 120$]^{42}$.

\footnotetext{
40 Sin embargo, es importante mencionar que estas ideas se originaron en la perspectiva de la tradición judeo cristiana (Ver Knox, 2001, p. 153)

41 SABATÉ MARTÍNEZ, Ana; RODRIGUEZ MOYA, Juan Maria; DIAZ MUÑOZ, María Ángeles. Mujeres, Espacio y Sociedad: Hacia una Geografía del Género. Madrid: Editorial Síntesis, 1995.

42 SABATÉ MARTÍNEZ, Ana; RODRIGUEZ MOYA, Juan María; DIAZ MUÑOZ, María Ángeles. Mujeres, Espacio y Sociedad: Hacia una Geografía del Género. Madrid: Editorial Síntesis, 1995.
}

148 Jorge Andrés Rivera - Magda Liliana Pérez 
Durante la década de los 80's la ecología política aporto un sustento teórico al activismo ambiental (movimientos verdes) los cuales buscaban como objetivo de lucha el reconocimiento de unas profundas modificaciones globales en el ambiente inducidas por el hombre. En este contexto aparecieron a mediados de esta década una serie de trabajos sobre la agricultura, industrialización, urbanización y otros fenómenos geográficos comparables, que intentaron vincular la nueva cultura ambientalista de carácter político con los análisis propios de la economía política ${ }^{43}$.

Otro tipo de concepciones y enfoques ambientalistas que tiene menor incidencia por parte de la geografía son el ecocentrismo y tecnocentrismo. Estos enfoques confirman que el movimiento ambiental abarca un amplio rango de intereses y objetivos basados en el examen y comprensión de las relaciones entre la naturaleza y procesos sociales.

El ecocentrismo es asociado con una visión no utilitaria del mundo natural, bioética, antimaterialista, que rechaza la noción de ciencia "objetiva", teniendo poca fe en el tecnicismo. A partir de esta visión se ha constituido el ecologismo ultraconservacionista, movimiento con un alto contenido romántico sobre la naturaleza, resaltando su armonía y los limites que ésta impone a los procesos económicos y sociales, y a la propia supervivencia de la especie humana.
Frecuentemente esta corriente ideológica ignora el carácter y dinámica de los procesos sociales y su incidencia contradictoria sobre las pretensiones conservacionistas y existencialistas de los propios abanderados.

En contraste, el tecnocentrismo ve incrementado el bienestar material como ideal universal, alcanzable a través de un adecuado manejo de los recursos; la visión de los tecnocentrístas es un mundo en el cual la ciencia y la tecnología tienen un valor gratuito, con un potencial infinito que pone los recursos naturales al servicio de los seres humanos. De esta manera, la institucionalización del cambio acelerado y la fe ciega en el progreso continuo han fortalecido la ideología de la omnipotencia absoluta de la sociedad sobre las fuerzas de la naturaleza y la creencia de que la humanidad tiene y tendrá cada vez mayores niveles de bienestar. Esta ideología oculta los limites de este progreso, esconde la esencia del cambio y minimiza fenómenos socialmente negativos asociados con él.

Actualmente la Sociedad de Filosofía y Geografía (Society for Philosophy and Geography, SPG), creada en 1994 con el propósito de unir esfuerzos e intercambiar puntos de vista en tópicos de mutuo interés, ha definido la discusión entorno a la teoría o epistemología ambiental como área temática de gran importancia. En la década de los 80 estas disciplinas habían demostrado un marcado

43 KNOX, Paul; MARSTON, Sallie. Human Geography: Places and Regions in the Global Context. New Jersey: Prentice Hall, 2001 
incremento en el interés por lo ambiental, pero cada una lo abordaba desde diferentes perspectivas. Muchos estudiosos de la ética ambiental aprecian la aproximación de síntesis de los geógrafos y han encontrado que su filosofía se ha fortalecido a través del análisis de trabajos geográficos. Por su parte los geógrafos, han estado deseando ampliar su objeto de estudio más allá de la descripción y explicación de la tierra como hogar de la especie humana, poniendo énfasis en evaluar críticamente las bases normativas de la preservación ambiental.

El geógrafo Bryan Norton ${ }^{44}$ ha mostrado que la ética ambiental ha sufrido de una inhabilidad para producir teorías que hayan ayudado significativamente en la creación de unas mejores políticas ambientales. Este autor afirma que la construcción de una teoría ambiental no debería ser soportada únicamente sobre las ideas de una sola disciplina. El ambientalista Noruego Sigmund Kvaloy ${ }^{45}$ reflexiona sobre esto de mejor manera cuando argumenta que las personas dedicadas a desarrollar teorías ambientales deberían esforzarse por ser tan amplios en alcances y visiones como lo son a la hora de luchar por la vida de los ecosistemas y la sociedad humana actual. De esta forma es evidente la necesidad de abordar el problema ambiental desde una aproximación interdisciplinaria, [LIGHT, SMITH, 1997].

No obstante, el interés de la asociación geográfica y filosófica se ha centrado principalmente entorno a los problemas generados por la ambigüedad en el significado del concepto "ambiente". Sobre la obra de Mead y Heidegger "Explorando la ética y la teoría del espacio, lugar y ambiente", Eliza Steelwater asegura que estos conceptos y sus significados han sido siempre objeto de negociación. Con base en el trabajo de Martín Heidigger y George Herbert Mead, esta autora insiste que cualquier ética ambiental debe estar relacionada con el mundo fenomenológico de la experiencia. En contraposición, la obra de Matthew Gandy sobre "Ecología, Modernidad y legado intelectual de la escuela de Frankfurt" intenta situarse aparte de las visiones más tradicionales. El es renuente a separar las fronteras entre los humanos y el mundo natural. Influenciado por la tradición de la teoría crítica alemana, y particularmente por escritores contemporáneos como Jurgen Habermas y Ulrich Beck, Gandy argumenta la necesidad de una ética soportada en una racionalidad comunicativa. Afirma que los problemas actuales no serán resueltos reformando la relación entre la sociedad y el mundo natural; particularmente si la responsabilidad por estas reformas están

\footnotetext{
4 NORTON, Bryan. Integration or Reduction: Two Approaches to Environmental Values. London: Routledge Press, 1996.

45 KVALOY, Sigmund. Complexity and Time: Breaking the Pyramid's Reign, in Wisdom in the Open Air.The Norwegian Roots of Deep Ecology. Minneapolis: University of Minnesota Press, 1993.
}

150 Jorge Andrés Rivera - Magda Liliana Pérez 
en manos de los expertos. En conclusión asegura que la ciencia es la fuente de los nuevos problemas ambientales como también de sus soluciones, [LIGHT, SMITH, 1997].

Finalmente, es importante mencionar que los geógrafos junto a los sociólogos, los sicólogos y los expertos en ciencias políticas han sido agentes activos e importantes en el debate ambiental, y han hecho notables contribuciones para el entendimiento de los orígenes, evolución e ideologías del movimiento ambiental moderno.

\section{Problemas ambientales}

Haciendo referencia al tema ambiental y en particular a los problemas geográficoambientales, Peet y Emel (1989), argumentaron que esta temática se originó como respuesta a un desmedido modo de vida con respecto a los altos niveles de consumo. De igual forma, estos autores sostienen que a los geógrafos les hace falta mayor compromiso con las verdaderas soluciones a esta problemática, es así como incitan a buscar en los aspectos y causas estructurales primarias, como ha mantener una mayor dedicación e inserción tanto en las investigaciones como en las acciones políticas de mayor relevancia. Sin embargo, existen excepciones notorias a esta generalización (Sayer, 1979; Pepper, 1984; Redclift, 1987), pero pese a ellas es obvio que profesionales de otras disciplinas, en especial los ecologistas (Gorz, 1979) e incluso los economistas (Turner, 1988), han hecho aportes importantes a un campo que a comienzos del siglo XX se consideraba crucial en la investigación y enseñanza geográficas, [Unwin, 1992].

De otro lugar, es necesario construir un interés interdisciplinario con relación a las cuestiones ambientales ya que para realizar el análisis del uso de los recursos y las estrategias de gestión, es ineludible comprender fenómenos y prácticas sociales muy diferentes. Según Rees (1989), la comprensión de los problemas relacionados con la explotación de recursos y el desarrollo de políticas de gestión precisa el examen de sistemas físicos, procesos económicos, organizaciones sociales, estructuras jurídicas y administrativas, e instituciones políticas.

En particular la geografía dentro de su construcción epistemológica define como fenómenos o problemas ambientales que ameritan un examen geográfico, entre otros; el cambio climático, deforestación, perdida de biodiversidad, deterioro de los suelos, reducción de los recursos hídricos y deterioro de los recursos costeromarinos, contaminación por residuos, emisiones y desechos sólidos, etc.

\subsection{Cambio Climático}

Con la transformación de los paisajes rurales a paisajes suburbanos y urbanos ocurren dramáticos cambios en los climas locales y regionales. Por ejemplo, el calentamiento y secamiento urbano ha sido medido y simulado por décadas por los geógrafos (Arnfield, 1982; Grimmond 
\& Oke, 1995). Estos modelos han empezado a prever los efectos de la urbanización sobre el clima. Las medidas indican que la evapotranspiración es aún mayor que la esperada en muchas áreas residenciales en las ciudades, debido a la irrigación de la vegetación plantada. En otras partes de estas, la evapotranspiración tiende a ser mucho menor a medida que la energía disponible calienta principalmente las fábricas urbanas.

Otro punto de investigación en el cambio ambiental involucra la reconstrucción de los patrones de perturbación recientes y de los procesos ecosistémicos sobre bosques, arbustales y comunidades de desierto a través de un cuidadoso análisis de campo e histórico (Veblen \& Lorenz, 1988). La disciplina también está abordando la perturbación y cambio sobre grandes escalas de tiempo a través del análisis de los sedimentos de los lagos de diferentes ecosistemas (Horn, 1993).

La geografía también ha centrado su atención sobre la naturaleza misma del cambio y las transiciones entre diferentes cambios de estado. Igualmente, existe un interés creciente sobre los flujos de energía y masa a través de la superficie del sistema terrestre como una forma de entender la estructura subyacente al cambio ambiental. Las investigaciones geográficas exploran dichos cambios a escalas de tiempo que fluctúan desde menos de un año a cientos de miles de años.
A escalas de tiempo de décadas a siglos, el trabajo geográfico esta relacionado principalmente con la documentación de los cambios en los sistemas de la superficie terrestre y evaluar las causas subyacentes. Por ejemplo, un tema de estudio involucra la reconstrucción de las dimensiones históricas de los glaciares a través de fotografías y muestreos para evaluar el cambio en el clima regional.

\subsection{Deforestación}

Un importante centro de atención del trabajo geográfico reciente han sido las variaciones en la naturaleza, recurrencia y consecuencias bióticas de las perturbaciones humanas y naturales tales como fuegos, tumba de árboles, clareo de bosques (Vale, 1982). Estas investigaciones dan un conocimiento esencial para desarrollar sistemas para preservar la biodiversidad a escalas locales, regionales y globales (Savage, 1993). El problema de la deforestación ha generado la pérdida de la biodiversidad por disminución del hábitat, la degradación de los suelos, el incremento de la escorrentía y del transporte de sedimentos en los cursos de agua, la reducción de las aguas subterráneas y los efectos adversos sobre el ciclo hidrológico.

El origen principal de la deforestación es la sustitución del bosque por sistemas agrícolas y ganaderos. El corte de madera para leña, los aprovechamientos para fines industriales y los incendios, plagas y enfermedades, contribuyen a la deforestación en menor proporción. 


\subsection{Pérdida de Biodiversidad}

Sus principales causas son la pérdida de hábitat por deforestación, la debilidad en la protección de las áreas silvestres, el aprovechamiento no sustentable de flora y fauna y la contaminación de fuentes de aguas. Los geógrafos estudian la distribución de especies de plantas y animales y como estas distribuciones son modeladas por condiciones locales y regionales -incluyendo la actividad humana- $y$ por la migración influenciada por los hombres y la selección (Sauer, 1988). La geografía también tiene una amplia tradición en el estudio de los determinantes humanos y no humanos de la biodiversidad tanto en paisajes 'naturales' como agrícolas.

El estrés por humedad, resultado de la escasa precipitación y de la rápida escorrentía impide, a su vez, la recuperación de la vegetación, lo cual conduce a una erosión acelerada y a un ciclo de retroalimentación positivo que ulteriormente degrada más tanto el suelo como la vegetación.

\subsection{Deterioro de los Suelos}

La discontinuidad del ciclo hidrológico, el acarreo de sedimentos, la pérdida de fertilidad de los ecosistemas y la pérdida de suelos, son algunas de las causas y de los efectos del deterioro de los suelos. La geógrafa Carol Harden ${ }^{46}$ ha estudiado la erosión del suelo en Norte, Centro y Suramérica: analizando cuando y donde ocurren, cuanto afecta y es afectada por los lugareños, y como el suelo erodado se mueve a través del paisaje para ser arrastrado por los ríos o ser atrapado en reservorios.

La investigación de Harden demostró que los caminos son los componentes generadores de escorrentía más activos de muchos paisajes tropicales y templados, produciendo escorrentía más rápidamente que otras superficies bajo el mismo evento de lluvia, generando escorrentía bajo lluvias que son muy ligeras para producir escorrentía en otras partes y extienden efectivamente la red de drenaje. El trabajo de Harden argumenta que los caminos afectan profundamente los procesos geomórficos/ erosionales y por lo tanto deben ser incorporados a los modelos hidrológicos y de erosión de suelos.

\subsection{Reducción de los Recursos Hídricos y Deterioro de los Recursos Costero-Marinos}

Otro centro importante de atención del trabajo geográfico a estas escalas de tiempo se relaciona con el impacto de los asentamientos humanos sobre los sistemas fluviales (Kesel 1992), los efectos de la urbanización sobre la escorrentía hídrica y de sedimentos a los ríos. Algunas cuencas hidrográficas, debido a la pérdida de cobertura forestal en zonas de recarga, han visto alterado su ciclo hidrológico, lo que provoca grandes avenidas e inundaciones en

$\overline{46}$ HARDEN, Carol. Andean Soil Erosion. National Geographic Research and Exploration, 1991. 
época de lluvia y disminución de caudales en época seca.

En cuanto al deterioro de los recursos costero-marinos, este se manifiesta en la explotación desordenada de los recursos pesquero-marinos, pérdida de bosque manglar y contaminación y sedimentación de las fuentes de agua, a causa del deterioro de los recursos, la sedimentación en los ríos por procesos de erosión, contaminación de los ríos por agroquímicos, habilitación de tierras para la agricultura, ganadería y/o salinas en zonas de bosque manglar, consumo de leña proveniente de bosque manglar para producción de sal y para consumo doméstico-energético; producción de postes, madera para construcción y tutores para actividades agrícolas.

\subsection{Contaminación por Residuos, Emisiones Atmosféricas y Desechos Sólidos}

Las actividades industriales y agroindustriales normalmente están ubicadas en áreas de influencia urbana municipal y vierten sus aguas residuales, sin tratamiento alguno, a los colectores sanitarios, pluviales o combinados. Cuando no hay acceso a un sistema de alcantarillado, los efluentes son lanzados sin tratamiento a los cuerpos de agua receptores. Adicionalmente, en los paisajes urbano-industriales existen multitud de focos contaminantes: los vehículos de motor, las calefacciones domésticas, los humos industriales, las alcantarillas, la combustión de basuras, etc; que producen sustancias peligrosas para la salud humana.

En particular, existen elementos globales que por su trascendencia e impacto, son identificados por los geógrafos humanos, como restrictivos para la conservación del medio ambiente y la utilización adecuada y sostenible de los recursos naturales. Algunos de ellos son:

- Desarrollo de políticas macroeconómicas que no consideran los impactos ambientales.

- El mercado no asegura siempre el uso y manejo adecuado del medio ambiente, porque los beneficios se manifiestan regularmente sólo a largo plazo.

- Tendencias migratorias de la población hacia áreas de vocación forestal o de alta concentración poblacional.

- Falta de conciencia individual y colectiva sobre la dimensión real del problema poblacional.

\section{Conclusiones}

A pesar de los esfuerzos realizados en la disciplina geográfica por unificar la geografía física y humana en función de una interpretación de la dialéctica sociedad-naturaleza, ha sido mínimo el logro alcanzado, debido a que la primera ha mantenido como objetivo principal de su desarrollo la explicación de los proceso físicos presentes y pasados que ocurren en el medio, esto se ha conseguido a

154 Jorge Andrés Rivera - Magda Liliana Pérez 
través de experimentaciones rigurosas, construcción de modelos y verificación de hipótesis, y la medición de su éxito ha dependido de la exactitud entre las predicciones expresadas, como por ejemplo en fórmulas matemáticas y las observaciones empíricas de la realidad; mientras la segunda ha orientado las investigaciones sobre el medio ambiente desde puntos de vista muy diferentes; algunos se han centrado en la búsqueda histórico-hermenéutica de la comprensión e interpretación, tratando de examinar el significado humano del paisaje. Otro enfoque adoptado por la geografía humana se ha basado en la conceptualización marxista de las relaciones entre la sociedad y la naturaleza; al mismo tiempo se han planteado diferentes vínculos entre hombre-medio, haciendo hincapié en la primacía de la sociedad humana sobre la naturaleza (posibilismo), y en oposición un punto de vista que sugiere que la sociedad humana es esencialmente un producto de fuerzas ambientales (determinismo).

La geografía ha definido como uno de los objetos de estudio más importantes dentro de la disciplina el análisis de la relación entre la sociedad y el medio. En este sentido un aporte fundamental es la síntesis como categoría de dominio interpretativo de todos los procesos comprometidos en dicha relación en su dimensión espacial; contribuyendo de manera significativa al avance del conocimiento ambiental en general y del manejo de ambientes específicos en territorios particulares.
La especificidad del trabajo geográfico en la resolución de problemas ambientales es notorio en el manejo de las escalas de observación. La geografía trabaja con ambas escalas, la temporal y la espacial. La disciplina ha estado profundamente enfocada en las interdependencias entre escalas como categoría de análisis de los problemas ambientales. La identificación de la escala a la cual un fenómeno particular exhibe sus máximas variaciones, proporciona importantes ideas en la resolución de los problemas acaecidos en la relación hombre-medio. Por ejemplo, el análisis espectral de los cambios de temperatura, revela que la escala geográfica en la cual es máxima, pude provenir de influencias relativas de microclimas, masas de aire, circulación global de patrones de temperatura. De igual modo, el enfoque de escalas habilita a los geógrafos para analizar el impacto de los cambios globales en eventos locales y el impacto de eventos locales en cambios globales.

El aporte de la geografía en el análisis de las dinámicas socio-ambientales se basa en el estudio de la relación entre la población, su base social y las fuentes ambientales que la soportan; enfocándose esencialmente en la naturaleza de esta relación, analizándola desde un contexto contemporáneo y local hasta procesos históricos y globales. Dentro de este estudio, los geógrafos están involucrados tanto en la recolección de la información hasta el análisis e identificación de las conexiones entre cambios en la población, el medio ambiente y las respuestas y reacciones sociales. 
El origen de la preocupación y los enfoques ambientales ha cambiado de perspectiva en tanto se ha advertido que su definición como problemática y tema crítico de interés internacional se ha tenido en cuenta en su verdadera dimensión hasta el momento en que se ha convertido en una amenaza legítima para el desarrollo normal de los países industrializados, generando un impacto negativo en su calidad de vida y bienestar. De esta forma se ha superado la idea vendida por occidente, que enunciaba una posición bioética y de compromiso intergeneracional sustentada en su temor por el creciente deterioro del medio ambiente en la reducción de recursos para sostener la población humana actual y futura, así como todas las demás formas de vida.

Los geógrafos trabajan en las interrelaciones entre diferentes procesos físicos y dinámicas sociales. Dentro de estos ámbitos o dominios de síntesis la geografía estudia cómo y por qué el hombre interviene en los cambios ambientales, así como también en las consecuencias para la humanidad de los cambios del medio natural. De la misma manera, los geógrafos han reconocido en estudios de percepción, el reconocimiento simbólico de las referencias de los elementos y la imágenes de los paisajes en su contexto social, histórico, geográfico y cultural. 


\section{Bibliografía}

ARNFIELD, A. J. An Approach to the Estimation of the Surface Radiative Properties per Radiation Budgets of Cities. Physical Geography, 1982.

BONILLA GODOY, Jaime. Notas de clase. sobre aproximaciones conceptuales a los problemas geográfico ambientales. Bogotá, 2002.

BRU I BISTUER, J. Medi Ambient I Equitat: la Perspectiva del Génere. Documents d'Anàlisi Geográfica, 1993.

CAPEL, Horacio. Filosofía y Ciencia en la geografía contemporánea. Ed. Barcanova. 1981.

CAPEL, Horacio; Urteaga Luis . Las nuevas geografías. Madrid. Ed. Salvat. 1985.

CLARK, John. The dialectical Social Geography of Elisée Reclus. Ed. Rowman \& Littlefield Publishers.

FLOREZ, Antonio; THOMAS Javier. La teoría General de Sistemas. Cuadernos de Geografía. Vol. IV, No.1-2, 1993.

FLOREZ, Antonio; FRANCOA., Maria Cristina; MONTAÑÉS GÓMEZ, Gustavo; DE MORENO, Elsa; TORRES DE CÁRDENAS, Rosa. Geografía y Ambiente: Enfoques y Perspectivas. Ediciones Universidad de la Sabana. 1997.

GORZ, A. Ecology and Politics. Boston: South End Press, 1979.

GLACKEN, Clarence. Traces on the Rhodian Shore. Nature and Culture in Western Thought from ancient times to the end of the Eighteenth Century. Berkeley: University of California Press. 1967.

GÓMEZ MENDOZA, Josefina; MUÑOZ JIMÉNEZ, Julio; ORTEGA CANTERO, Nicolás. El pensamiento geográfico. Ed. Alianza Universidad. 1982.

GREGORY, Derek. Ideología, Ciencia y Geografía Humana. Barcelona: Ediciones Oikos-tau, 1984.

GREGORY, Derek; MARTIN, Ron; SMITH, Graham. Human Geography. Society, Space, and Social Science. University of Minnesota: Press Minneapolis, 1994.

GRIMMOND, C. S. B.; OKE, T. R. Comparison of Heat Fluxes from Summer Time Observations in the Suburbs of Four North American Cities. Journal of Applied Meteorology, 1995. HANSON, Susan. Ten geographic ideas that chanced the world. University Press., New Brunswick, New Jersey, 1997.

HARO, Juan. Calidad y conservación del medio ambiente. Ed. Cincel, 1983. 
HARVEY, David. Teorías, Leyes y Modelos en Geografía. Madrid. Ed. Alianza Editorial. 1983. HARVEY, David. Justice, Nature and the geography of difference. Blackwell Publishers Editor, 1996.

HORN, S. P. Postglacial Vegetation and Fire History in the Chirripó Páramo of Costa Rica. Quaternary Research, 1993.

HUNTINGTON, Ellsworth. Civilization and Climate. New Haven: Yale University Press, 1915. HUNTINGTON, Ellsworth. The Human Habitat. United States of America: Plimpton Press, 1929.

JOHNSTON, R.J. The Challenge for Geography. A Changing World: A Changing Discipline. Ed. Blackwell, 1993.

KESEL, R. H. An Approximation of the Sediment Budget of the Lower Mississippi River Prior to Major Human Modification. Earth Surface Processes and Landforms, 1992.

KNOX, Paul; MARSTON, Sallie. Human Geography: Places and Regions in the Global Context. New Jersey: Prentice Hall, 2001.

LIGHT, Andrew; SMITH, Jonathan. Philosophy and Geography I: Space, Place, and Environment Ethics. Ed. Rowman \& Littlefield Publishers, 1997.

NATIONAL RESEARCH COUNCIL. Rediscovering Geography. 1997.

PEET, Richard. Modern Geographical Thought. Blackwell Publishers Editor, 1998.

PEPPER, D. The Roots of Modern Environmentalism. London: Croom Helm, 1984.

REES, J. Natural Resources Economy and Society. Basingstoke: Macmillan, 1989.

REDCLIFT, M. The production of Nature and the Reproduction of the Species. Antípode, 1987. REVISTA SCRIPTA NOVA. Revista Electrónica de Geografía y Ciencias Sociales. Barcelona. 2000.

SAUER, J. D. Plant Migration: The dynamics of Geographic Patterning in Seed Plant Species. Berkeley University of California Press, 1988.

SAVAGE, M. Ecological Disturbance and Nature Tourism. Geographical Review, 1993.

SAYER, A. Epistemology and Conceptions of People and Nature in Geography, Geoforum, 1979.

SIMMONS, I. G. Changing the Face of the Earth: Culture, Environment, History. Oxford: Basil Blackwell, 1990.

TAYLOR, Thomas Griffith. Environment and Race: A Study of the Evolution, Migration, Settlement and Status of the Races of Man. London: Oxford University Press, 1927.

TURNER, R. K. Sustainable Environmental Management: Principles and Practice. London: Belhaven Press, 1988.

UNWIN, Tim. El Lugar de la geografía. Ediciones Cátedra, S.A, 1995.

VALE, T. R. Plants and People: Vegetation Change in North America. Washington D.C.: Association of American Geographers, 1982.

VIDART, Daniel. Filosofía Ambiental. Bogotá: Editorial Nueva América, 1986.

VEBLEN, T. T.; LORENZ, D.C. Recent Vegetation Changes Along the Forest/Steppe Ecotone of Northern Patagonia. Annals of the Association of American Geographers, 1988.

VIELZA DE ORY. Geografía general II. Ed Taurus. Madrid. 1984.

158 Jorge Andrés Rivera - Magda Liliana Pérez 